\title{
Nonlinear modal analysis of the SmallSat spacecraft
}

\author{
L. Renson, G. Kerschen \\ Space Structures and Systems Laboratory (S3L), Structural Dynamics Research Group \\ Department of Aerospace and Mechanical Engineering, University of Liège, Belgium
}

\section{A. Newerla}

European Space Agency (ESTEC), Noordwijk, The Netherlands

\begin{abstract}
Non-linear elements are present in practically all spacecraft structures. The assumption of a (quasi-)linear structure is nevertheless adequate for structural analyses and design verification purposes in those cases where these structural non-linearities are relatively weak or not substantially activated by the mechanical environments encountered during the launch or during ground testing. However, when significant non-linear effects in spacecraft structures are no longer negligible then linear modal analysis will not be able to handle non-linear dynamical phenomena in an adequate manner: the development of a non-linear analogue of linear modal analysis becomes an urgent and important issue. The objective of this paper is to show that nonlinear normal modes (NNMs) represent a useful and practical tool in this context. A full-scale spacecraft structure is considered and is modeled using the finite element method. Its NNMs are computed using advanced numerical algorithms, and the resulting dynamics is then carefully analyzed. Nonlinear phenomena with no linear counterpart including nonlinear modal interactions are also highlighted.
\end{abstract}

Keywords: nonlinear dynamics, modal analysis, nonlinear normal modes, space structure.

\section{INTRODUCTION}

Spacecraft structures are subjected to severe dynamic environments during the launch phase. In order to ensure the structural integrity of the spacecraft $(\mathrm{SC})$ and the payload $(\mathrm{PL})$ items minimum frequency requirements are usually defined for the SC in order to avoid dynamic coupling between the main frequency ranges of the launch vehicle (LV) excitations and the SC fundamental linear normal modes (LNMs), i.e. those modes where the effective modal masses are important. The launcher authority might however accept a non-compliance with the requirements in those cases where the SC eigenmode represents a "localized" dynamic effect with only a small effective mass involved.

From a linear point of view, these requirements avoid potentially disastrous coupling and energy exchanges between the LV and the SC. However, for nonlinear structures, this article will show on a representative SC structure developed by EADS Astrium, the SmallSat, that these requirements might not be sufficient. In particular, the excitation of global SC structure modes and PL modes involving local nonlinear effects is presented.

The paper is organized as follows. In Section 2, a brief review of nonlinear normal modes (NNMs) is achieved. In Section 3 the SC structure and its finite element model are described. The modeling of the nonlinearities is also discussed. In Section 4 , a linear modal analysis of the SC is performed and employed as an introduction to the nonlinear modal analysis of Section 6 . Section 5 shortly presents the algorithm used for the computation of nonlinear normal modes. Finally, Section 6 presents and discuss different NNMs of the structure. 


\section{REVIEW OF NORMAL MODES FOR NONLINEAR SYSTEMS}

A detailed description of NNMs and of their fundamental properties (e.g., frequency-energy dependence, bifurcations, and stability) is given in ${ }^{[1,2]}$ and is beyond the scope of this paper. For completeness, the main definition of a conservative NNM is briefly reviewed in this section.

The free response of discrete mechanical systems with $N$ degrees of freedom (DOFs) is considered, assuming that continuous systems (e.g., beams, shells, or plates) have been spatially discretized using the FE method. The equations of motion are

$$
\mathbf{M} \ddot{\mathbf{x}}(t)+\mathbf{K} \mathbf{x}(t)+\mathbf{f}_{\mathrm{nl}}\{\mathbf{x}(t)(t)\}=0
$$

where $\mathbf{M}$ and $\mathbf{K}$ are the mass and stiffness matrices, respectively; $\mathbf{x}$ and $\ddot{\mathbf{x}}$ are the displacement and acceleration vectors, respectively; $f_{n l}$ is the nonlinear restoring force vector.

Targeting a straightforward nonlinear extension of the concept of LNMs, Rosenberg defined an NNM motion as a synchronous periodic oscillation. This definition requires that all material points of the system reach their extreme values and pass through zero simultaneously and allows all displacements to be expressed in terms of a single reference displacement. At first glance, Rosenberg's definition may appear restrictive in two cases:

1. In the presence of internal resonances, an NNM motion is no longer synchronous, but it is still periodic. This is why an extended definition was considered in ${ }^{[2,3]}$; an NNM motion was defined as a (non-necessarily synchronous) periodic motion of the undamped mechanical system.

2. The definition cannot be easily extended to nonconservative systems. However, as shown in ${ }^{[2]}$, the damped dynamics can be interpreted based on the topological structure of the NNMs of the underlying conservative system, provided that damping has a purely parasitic effect.

For illustration, the system depicted in Figure 1 and governed by the equations

$$
\begin{aligned}
\ddot{x}_{1}+\left(2 x_{1}-x_{2}\right)+0.5 x_{1}^{3} & =0 \\
\ddot{x}_{2}+\left(2 x_{2}-x_{1}\right) & =0
\end{aligned}
$$

is considered. The NNMs corresponding to in-phase and out-of-phase motions are represented in the frequency-energy plot (FEP) of Figure 2. An NNM is represented by a point in the FEP, which is drawn at a frequency corresponding to the minimal period of the periodic motion and at an energy equal to the conserved total energy during the motion. A branch, represented by a solid line, is a family of NNM motions possessing the same qualitative features (e.g., in-phase NNM motion).

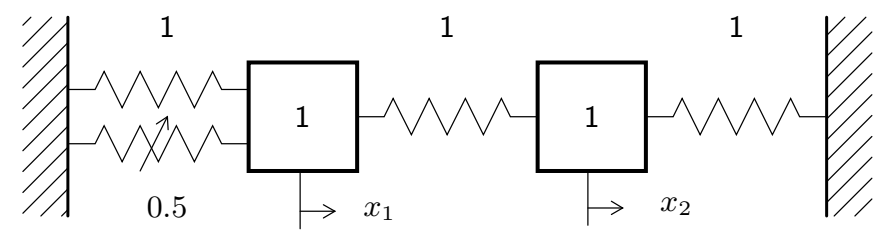

Figure 1: Schematic representation of the 2DOF system example.

\section{THE SMALLSAT SPACECRAFT AND ITS FINITE ELEMENT MODELLING}

The SmallSat structure has been conceived as a low cost structure for small low-earth orbit satellite ${ }^{[4]}$. It is a monocoque tube structure which is $1.2 \mathrm{~m}$ long and $1 \mathrm{~m}$ large. It incorporates eight flat faces for equipment mounting purposes, creating an octagon shape, as shown in Figure 3 (a). The octagon is manufactured using carbon fibre reinforced plastic by means of a filament winding process. The structure thickness is $4.0 \mathrm{~mm}$ with an additional $0.25 \mathrm{~mm}$ thick skin of Kevlar applied to both the inside and outside 


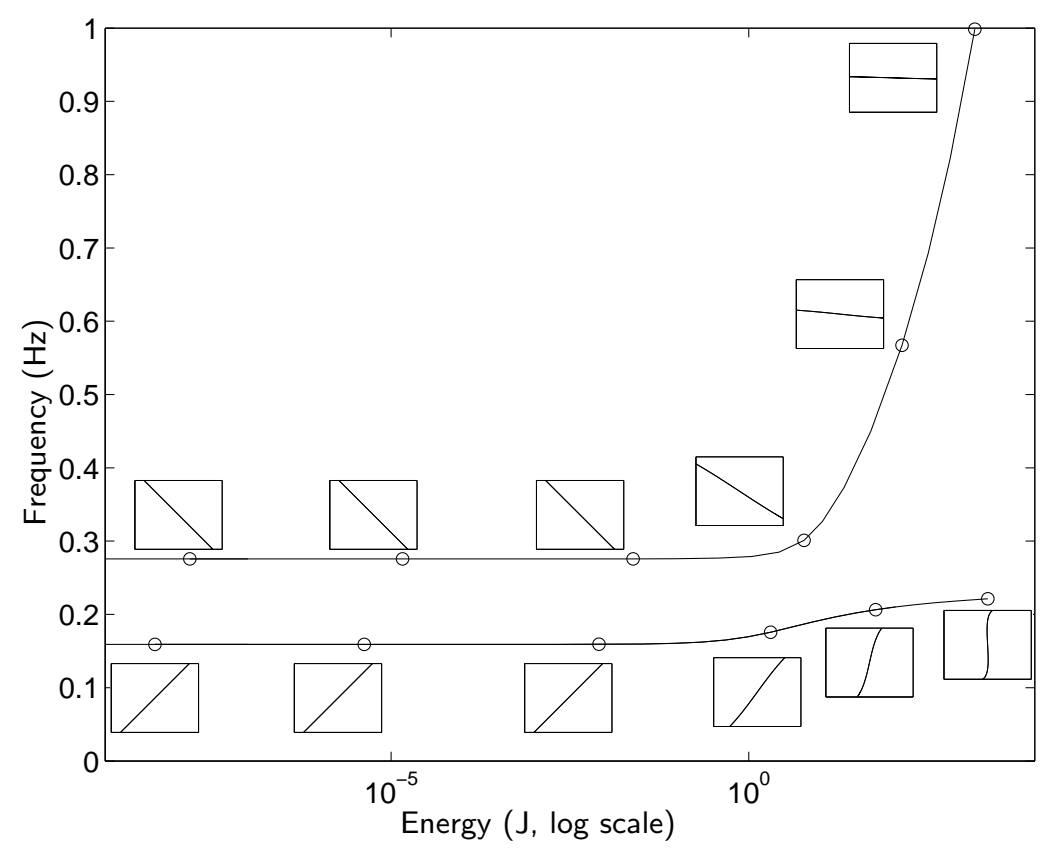

Figure 2: Frequency-energy plot of system (2). NNM motions depicted in the configuration space are inset.

surfaces to provide protection against debris. The interface between the spacecraft and launch vehicle is achieved through four aluminium brackets located around cut-outs at the base of the structure. The total mass including the interface brackets is around $64 \mathrm{~kg}$.

The SmallSat structure supports a telescope dummy composed of two stages of base-plates and struts supporting various concentrated masses; its mass is around $140 \mathrm{~kg}$. The telescope dummy plate is connected to the SmallSat top floor via three shock attenuators, termed SASSA (Shock Attenuation System for Spacecraft and Adaptator) ${ }^{[5]}$, the behaviour of which is considered as linear in the present study. The top floor is a 1 square meter sandwich aluminium panel, with $25 \mathrm{~mm}$ core and $1 \mathrm{~mm}$ skins. Finally, as shown in Figure 3 (c), a support bracket connects to one of the eight walls the so-called Wheel Elastomer Mounting System (WEMS) device which is loaded with an $8 \mathrm{~kg}$ reaction wheel dummy. The purpose of this device is to isolate the spacecraft structure from disturbances coming from reaction wheels through the presence of a soft interface between the fixed and mobile parts. In addition, mechanical stops limit the axial and lateral motion of the WEMS mobile part during launch, which gives rise to nonlinear dynamic phenomena. Figure 3 (d) depicts the WEMS overall geometry, but details are not disclosed for confidentiality reasons.

The Finite Element (FE) model in Figure 3 (b) was created in Samcef software and is used in the present study to conduct numerical experiments. The comparison with experimental measurements revealed the good predictive capability of this model. The WEMS mobile part (the inertia wheel and its cross-shaped support) was modeled as a flexible body, which is connected to the WEMS fixed part (the bracket and, by extension, the spacecraft itself) through four nonlinear connections, labeled NC 1-4 in Figure 3 (d). Black squares signal such connections. Each nonlinear connection possesses

- a nonlinear spring (elastomer in traction plus 2 stops) in the axial direction,

- a nonlinear spring (elastomer in shear plus 1 stop) in the radial direction,

- a linear spring (elastomer in shear) in the third direction.

The spring characteristics (piecewise linear) are listed in Table 1 and are displayed in Figure 3 (e). We stress the presence of two stops at each end of the cross in the axial direction. This explains the corresponding symmetric bilinear stiffness curve. In the radial direction, a single stop is enough to limit the motion of the wheel. For example, its $+x$ motion is constrained by the lateral stop number 2 while the connection $1-x$ limits the opposite $-x$ motion. The corresponding stiffness curves are consequently asymmetric. 

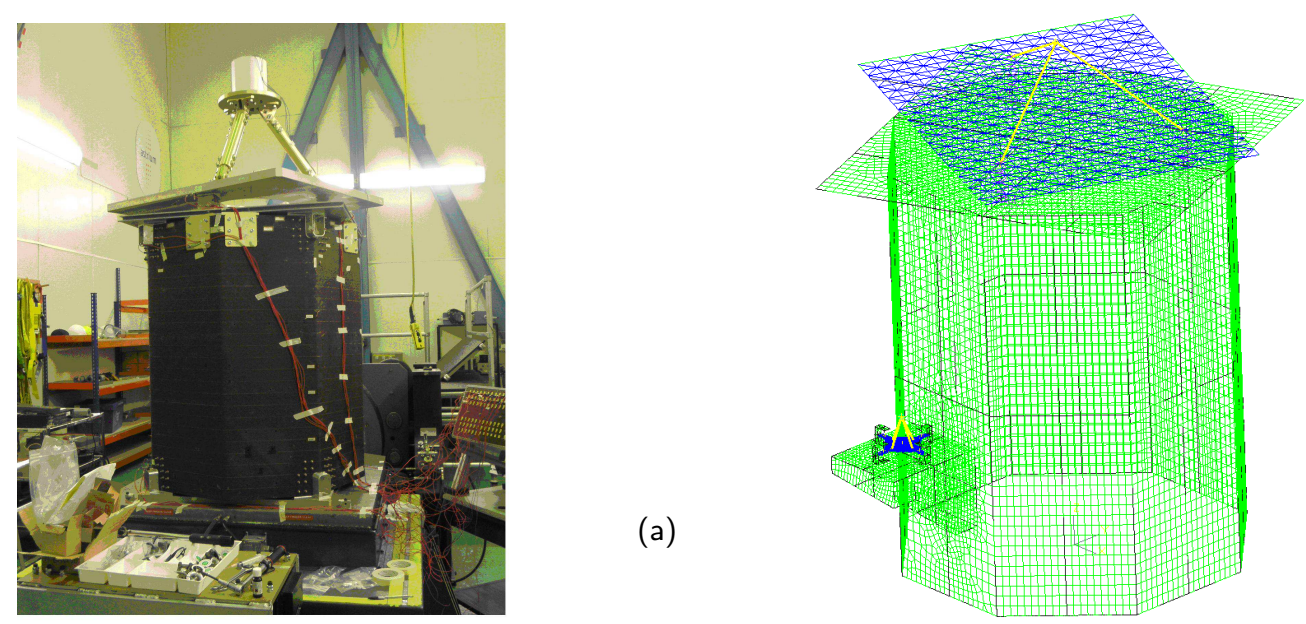

(b)
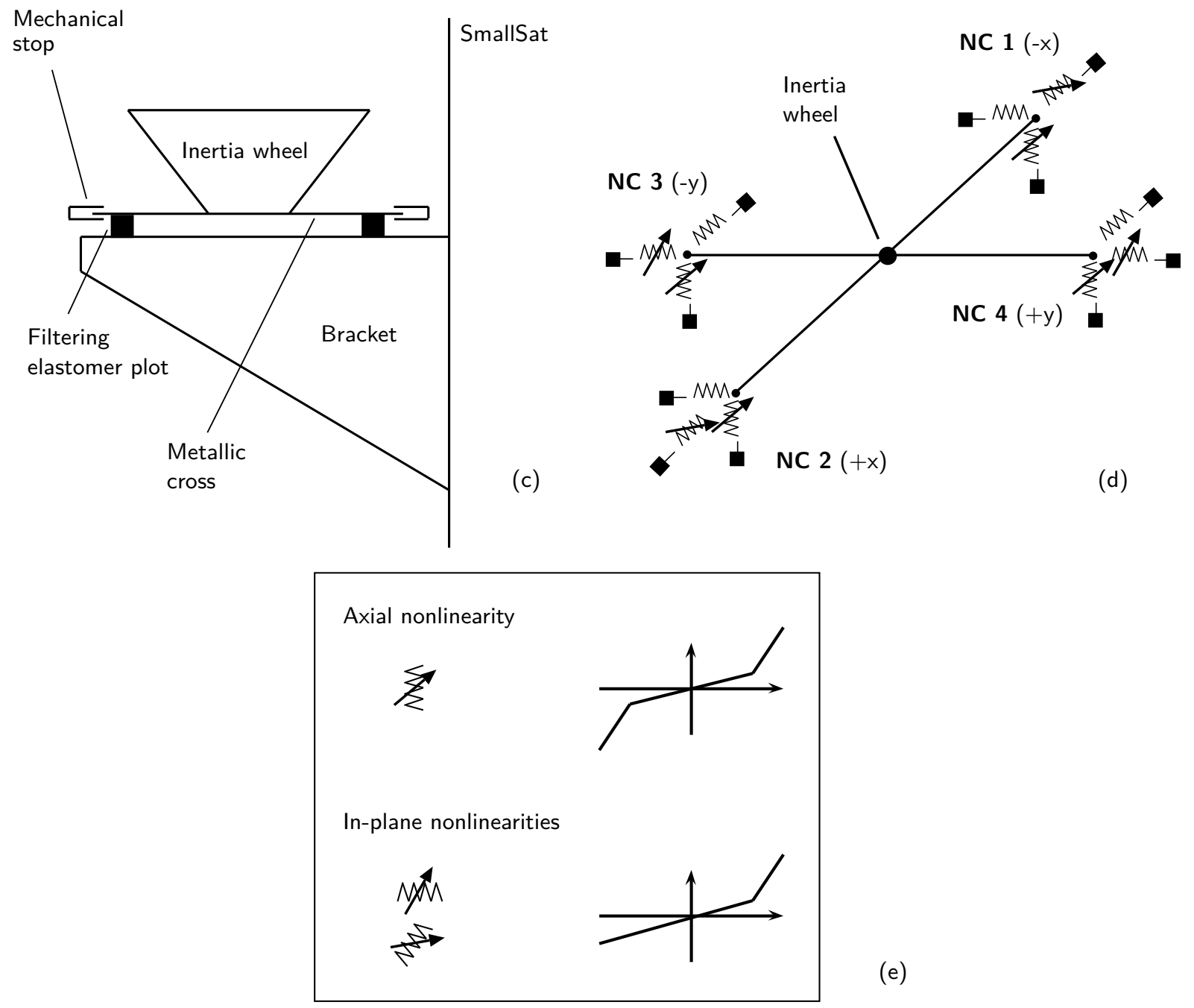

(e)

Figure 3: SmallSat structure. (a) real structure without the WEMS module; (b) finite element model; (c) WEMS module mounted on a bracket and supporting a dummy inertia wheel; (d) close-up of the WEMS mobile part (NC stands for nonlinear connection) and (e) graphical display of the nonlinear restoring forces. 


\begin{tabular}{|c|c|c|c|}
\hline Spring & Clearance & Stiffness of the elastomer plot & Stiffness of the mechanical stop \\
\hline Axial & $c_{\text {axial }}=1$ & 1 & 13.2 \\
Lateral & $c_{\text {radial }}=1.27$ & 0.26 & 5.24 \\
\hline
\end{tabular}

Table 1: Nonlinear spring characteristics (adimensional values for confidentiality).

\subsection{Nonlinearities modeling}

From a computational standpoint, the use of piecewise linear stiffnesses requires special numerical treatments, which increase the computational burden and complicate convergence processes. Therefore, piecewise behaviors are usually regularized.

One can avoid the introduction of piecewise-linear stiffnesses in replacing them by polynomials (e.g. using a single nonlinear term as in Equation (3)). Despite its simplicity, this approach has the disadvantage to consider a nonlinear behavior from the origin.

$$
F_{r}=k_{\operatorname{lin}} x+k_{\mathrm{n} 1} x^{n}
$$

In order to avoid such approximation, the regularization technique developed in this paper uses Hermite polynomials to smooth the transition between both linear stiffnesses. A regularization area $[a-\Delta, a+\Delta]$ is considered where $a$ is the transition point between linear regimes and where $2 \Delta$ is the size of the regularization area (Figure 4). This approach has the advantage to keep the restoring force behavior purely linear out of the regularization area (Figure 4).

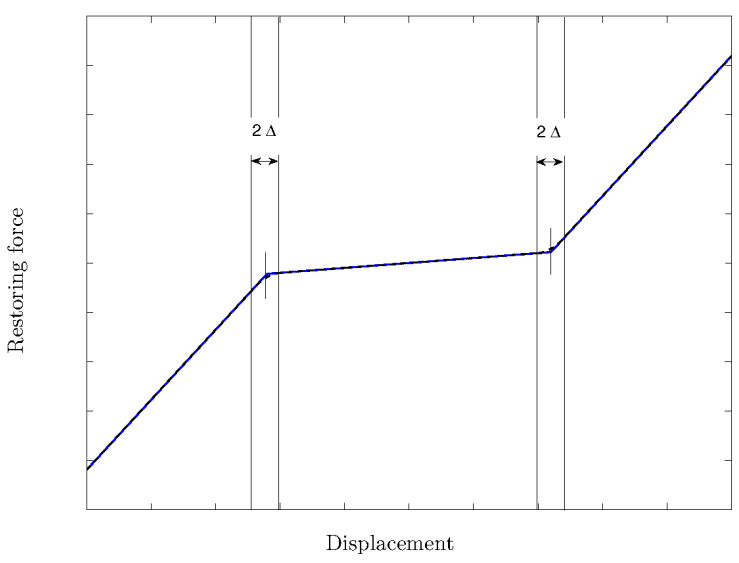

(a)

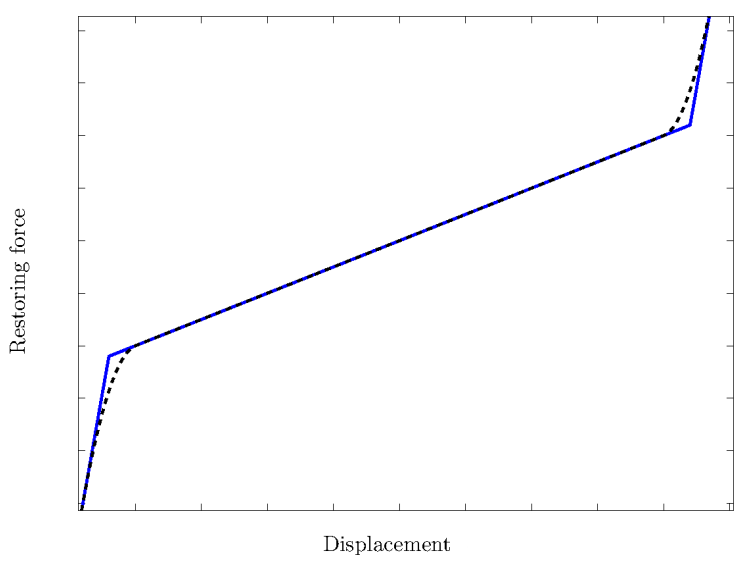

(b)

Figure 4: Piecewise-linear stiffness (-) and regularized stiffness (--). (a) Restoring force overview. (b) Zoom on regularization areas.

The nonlinear force is now given by Equation (4) and displayed in Figure 4.

$$
f_{\mathrm{nl}}(x)= \begin{cases}\operatorname{sign}(x)\left(k_{1} a+k_{2}(|x|-a)\right) & |x| \geq a+\Delta \\ p_{ \pm}(t(x)) & a+\Delta>|x|>a-\Delta \\ k_{1} x & a-\Delta \geq|x| \geq 0\end{cases}
$$

where $t(x)$ is defined by (5) and is used in the definition of the Hermite interpolation polynomials $p_{ \pm}(t(x))$ (Equation (6)).

$$
\begin{gathered}
t(x)=\frac{x-x_{k}}{x_{k+1}-x_{k}} \\
p_{ \pm}(t)=h_{00}(t) p_{k}+h_{10}(t)\left(x_{k+1}-x_{k}\right) m_{k}+h_{01}(t) p_{k+1}+h_{11}(t)\left(x_{k+1}-x_{k}\right) m_{k+1}
\end{gathered}
$$


where $p_{k}$ and $p_{k+1}$ are the values of the restoring force at the points $x_{k}$ and $x_{k+1} \cdot x_{k}=\operatorname{sign}(x)(a-\Delta)$ and $x_{k+1}=\operatorname{sign}(x)(a+\Delta)$. $m_{k}$ and $m_{k+1}$ are the values of the derivatives at the same points. For the piecewise-linear stiffness, $m_{k}=k_{1}$ and $m_{k+1}=k_{2}$. The $h_{i j}(t)$ are given by $(7)-(10)$.

$$
\begin{aligned}
& h_{00}(t)=2 t^{3}-3 t^{2}+1 \\
& h_{10}(t)=t^{3}-2 t^{2}+t \\
& h_{01}(t)=-2 t^{3}+3 t^{2} \\
& h_{11}(t)=t^{3}-t^{2}
\end{aligned}
$$

\section{LINEAR MODAL ANALYSIS}

Before the computation of NNMs (Section 6), a linear modal analysis is performed. The frequency range of interest for this study is $[0-100] \mathrm{Hz}$. Table 2 presents the resonance frequencies of the LNMs of the structure included in this frequency range. Among these LNMs, four are considered of particular interest for the nonlinear modal analysis of this paper. The first one is the first mode (Figure 5(a)) which presents a local WEMS motion with a concave trajectory about the axis $A$ (Figure 6). The second mode considered is the third LNM (Figure $5(\mathrm{~b})$ ) which again presents a local WEMS motion with convexe trajectory about $A$. The seventh LNM involving a SASSA mode with alternate compression of springs 1 and $2-3$ (Figure 7 ) is considered. This mode also includes a vertical motion of the WEMS. Finally, the ninth mode which presents a local SASSA mode without WEMS motion is considered.

\begin{tabular}{|c|c||c|c|}
\hline- & Frequency [Hz] & - & Frequency [Hz] \\
\hline \hline Mode 1 & 10.66 & Mode 11 & 43.30 \\
\hline Mode 2 & 11.00 & Mode 12 & 52.59 \\
\hline Mode 3 & 28.12 & Mode 13 & 71.36 \\
\hline Mode 4 & 28.38 & Mode 14 & 75.73 \\
\hline Mode 5 & 30.18 & Mode 15 & 80.44 \\
\hline Mode 6 & 30.50 & Mode 16 & 84.35 \\
\hline Mode 7 & 31.60 & Mode 17 & 90.52 \\
\hline Mode 8 & 32.65 & Mode 18 & 95.41 \\
\hline Mode 9 & 37.42 & Mode 19 & 101.56 \\
\hline Mode 10 & 38.26 & Mode 20 & 101.93 \\
\hline
\end{tabular}

Table 2: Summary of the linear normal mode frequencies.

\subsection{Reduced-order model}

As presented in Section 3, the finite element model contains more than 65000 dofs. It appears that the computation of the NNMs for such a large number of dofs is not currently feasible in a reasonable amount of time. Therefore, a reduced-order model (ROM) was created using the Craig-Bampton technique ${ }^{[6]}$.

This method consists in describing the system in terms of some retained DOFs and internal vibration modes. By partitioning the complete system in terms of $n_{R}$ remaining $\mathbf{x}_{R}$ and $n_{C}=n-n_{R}$ condensed $\mathbf{x}_{C}$ DOFs, the $n$ governing equations of motion of the global finite element model are written as

$$
\left[\begin{array}{ll}
\mathbf{M}_{R R} & \mathbf{M}_{R C} \\
\mathbf{M}_{C R} & \mathbf{M}_{C C}
\end{array}\right]\left[\begin{array}{c}
\ddot{\mathbf{x}}_{R} \\
\ddot{\mathbf{x}}_{C}
\end{array}\right]+\left[\begin{array}{ll}
\mathbf{K}_{R R} & \mathbf{K}_{R C} \\
\mathbf{K}_{C R} & \mathbf{K}_{C C}
\end{array}\right]\left[\begin{array}{c}
\mathbf{x}_{R} \\
\mathbf{x}_{C}
\end{array}\right]=\left[\begin{array}{c}
\mathbf{g}_{R} \\
\mathbf{0}
\end{array}\right]
$$

The Craig-Bampton method expresses the complete set of initial DOFs in terms of: (i) the remaining DOFs through the static modes (resulting from unit displacements on the remaining DOFs) and (ii) a certain number $m<n_{C}$ of internal vibration modes (relating to the primary structure fixed on the remaining nodes). Mathematically, the reduction is described by relation

$$
\left[\begin{array}{c}
\mathbf{x}_{R} \\
\mathbf{x}_{C}
\end{array}\right]=\left[\begin{array}{cc}
\mathbf{I} & \mathbf{0} \\
-\mathbf{K}_{C C}^{-1} \mathbf{K}_{C R} & \mathbf{\Phi}_{m}
\end{array}\right]\left[\begin{array}{c}
\mathbf{x}_{R} \\
\mathbf{y}
\end{array}\right]=\mathbf{R}\left[\begin{array}{c}
\mathbf{x}_{R} \\
\mathbf{y}
\end{array}\right]
$$




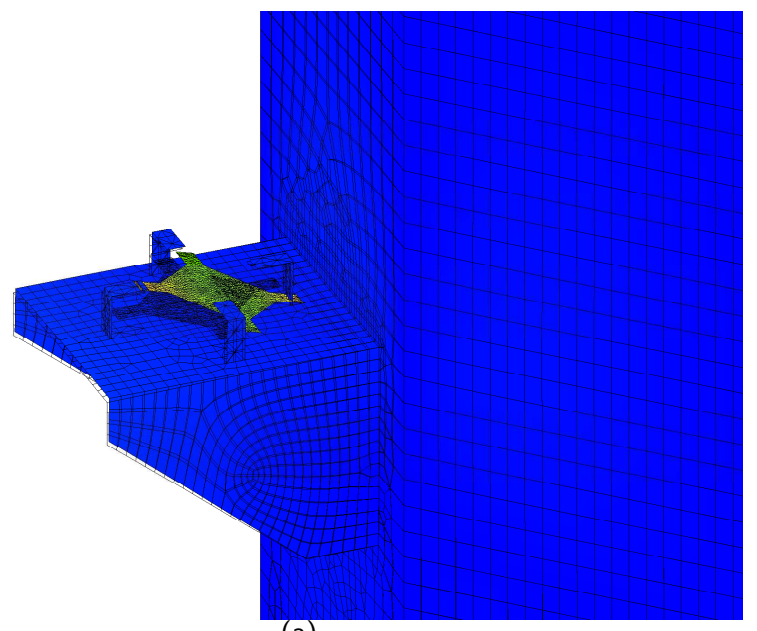

(a)

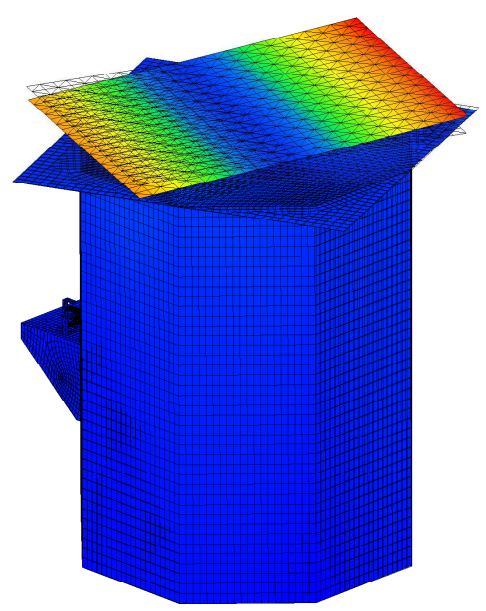

(c)

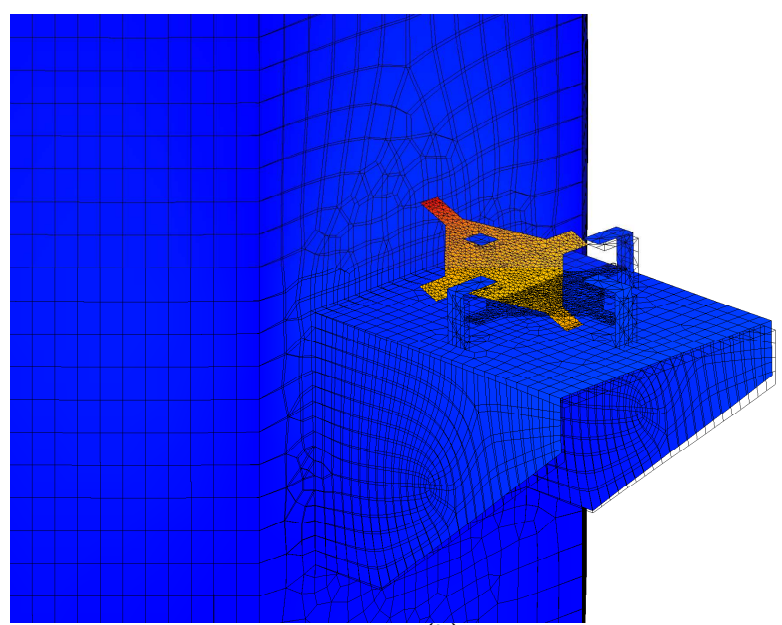

(b)

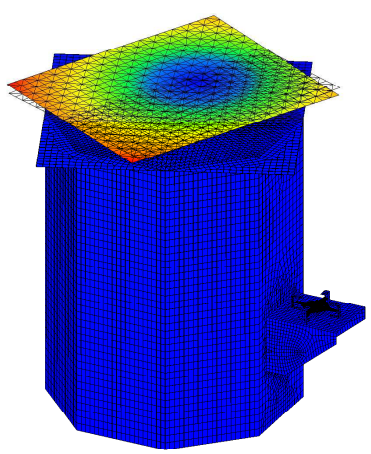

(d)

Figure 5: First (a), third (b), seventh (c), and ninth (d) LNM modal shapes of the SmallSat.

which defines the $n \times\left(n_{R}+m\right)$ reduction matrix $\mathbf{R}$. $\mathbf{y}$ are the modal coordinates of the $m$ internal linear normal modes collected in the $n_{C} \times m$ matrix $\boldsymbol{\Phi}_{m}=\left[\phi_{(1)} \ldots \phi_{(m)}\right]$. These modes are solutions of the linear eigenvalue problem corresponding to the system fixed on the remaining nodes

$$
\left(\mathbf{K}_{C C}-\omega_{(j)}^{2} \mathbf{M}_{C C}\right) \phi_{(j)}=\mathbf{0}
$$

The reduced model is thus defined by the $\left(n_{R}+m\right) \times\left(n_{R}+m\right)$ reduced stiffness and mass matrices given by

$$
\begin{aligned}
\overline{\mathbf{M}} & =\mathbf{R}^{*} \mathbf{M} \mathbf{R} \\
\overline{\mathbf{K}} & =\mathbf{R}^{*} \mathbf{K R}
\end{aligned}
$$

where star denotes the transpose operation. After reduction, the system configuration is expressed in terms of the reduced coordinates (i.e., the remaining DOFs and the modal coordinates). The initial DOFs of the complete model are then determined by means of the reduction matrix using relation 12 .

Table 3 summarizes the features of the different ROMs investigated. The eight nodes involved in the superelement definition are the minimum ones required to define the different nonlinearities of the WEMS.

Before proceeding to nonlinear analysis, the accuracy of the reduced-order linear model is assessed. To this end, the linear normal modes of the initial complete finite element model are compared to those predicted by the reduced model. The deviation between 


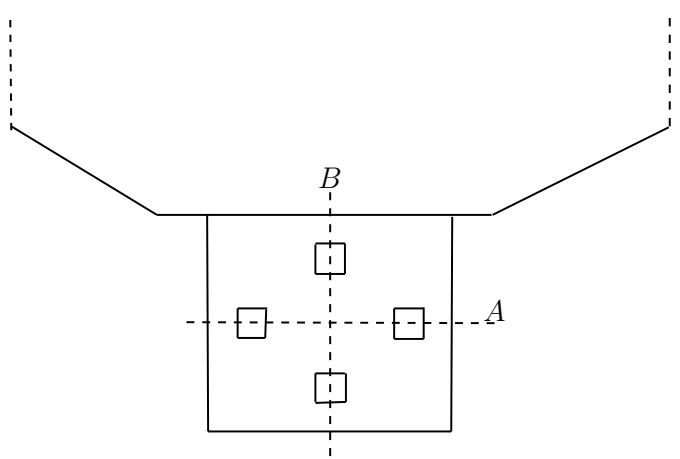

Figure 6: WEMS local axis.

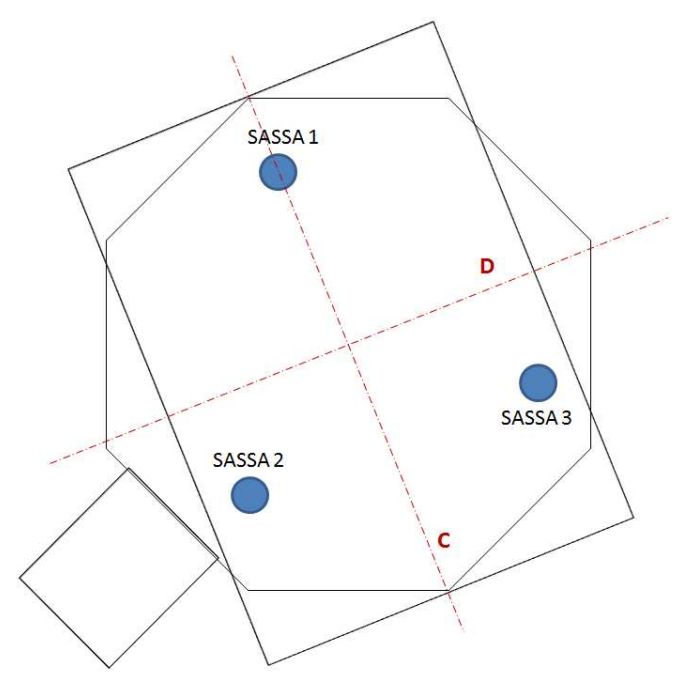

Figure 7: SASSA local axis

the mode shapes of the original model $\mathbf{x}_{(o)}$ and of the reduced model $\mathbf{x}_{(r)}$ is determined using the Modal Assurance Criterion (MAC)

$$
\mathrm{MAC}=\frac{\left|\mathbf{x}_{(o)}^{*} \mathbf{x}_{(r)}\right|^{2}}{\left|\mathbf{x}_{(o)}^{*} \mathbf{x}_{(o)}\right|\left|\mathbf{x}_{(r)}^{*} \mathbf{x}_{(r)}\right|}
$$

MAC values range from 0 in case of no correlation to 1 for a complete coincidence. The minimum correlation criteria are a maximum relative error on frequencies of $1 \%$ and MAC values above 0.9 in the frequency range $[0-200] \mathrm{Hz}$. Both criteria are displayed for each ROM in Figure 8 while Table 4 presents the frequency range accuratly covered by the different models.

ROMs with 100 and 500 internal modes both satisfy accuracy requirements. However the selection of the appropriate ROM for Section 6 is not trivial. A ROM with numerous internal modes provides the best chances to observe the modal interactions but this larger model increases the computation time. In addition, the number of modal interactions observed tends to become prohibitive and dramatically increases the computation time too. Therefore, in the following parts of this study, the reference ROM used for the computation of NNMs is "ROM85".

\section{NUMERICAL COMPUTATION OF NNMS}

The numerical method proposed here for the NNM computation relies on two main techniques, namely a shooting technique and the pseudo-arclength continuation method. A detailed description of the algorithm is given in ${ }^{[7]}$. 


\begin{tabular}{|c|c|c|}
\hline Model & Nodes & Internal modes \\
\hline ROM84 & 8 & 50 \\
ROM85 & 8 & 100 \\
ROM86 & 8 & 500 \\
\hline
\end{tabular}

Table 3: Features of the different reduced-order models created.
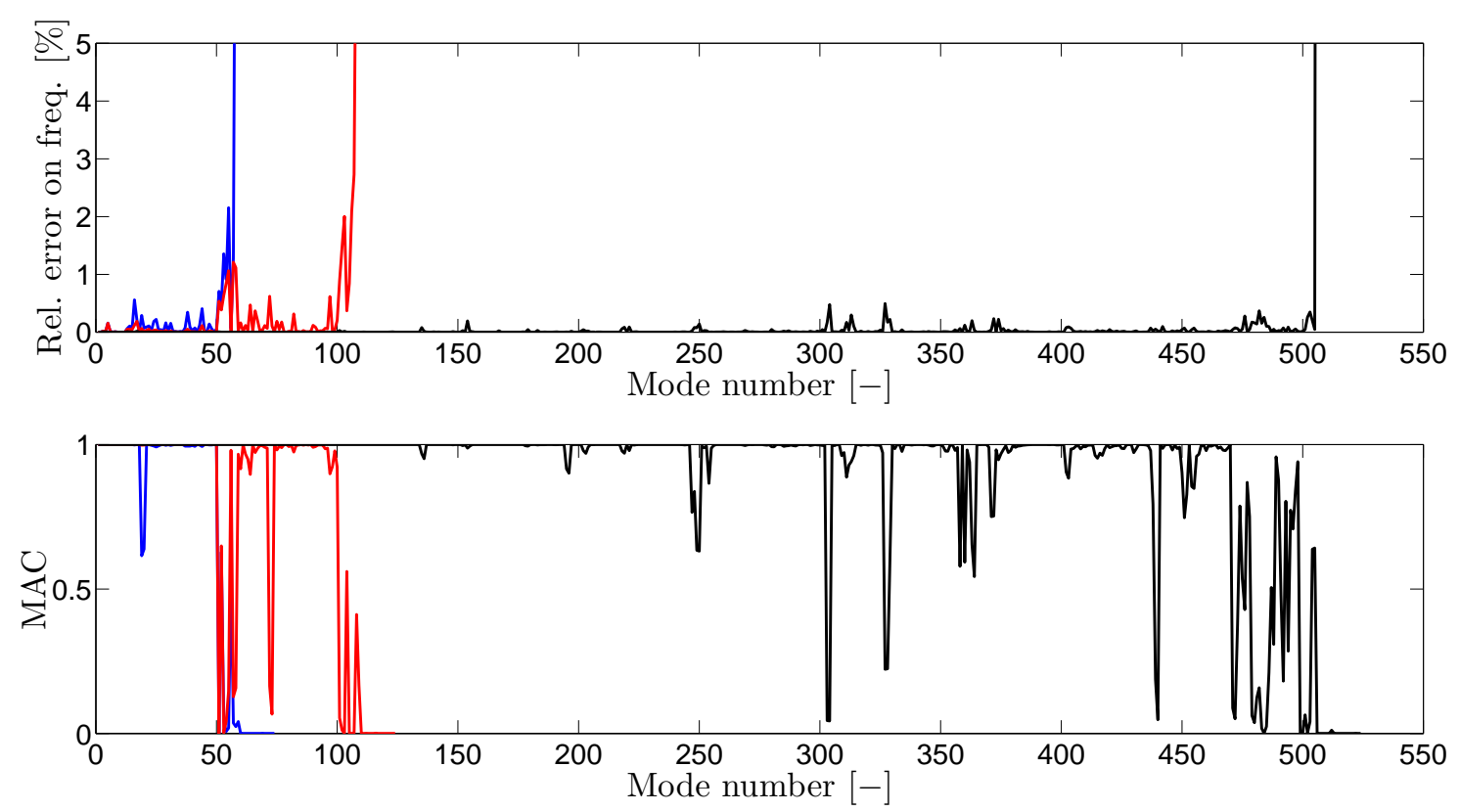

Figure 8: Relative error on frequencies and MAC for the different ROMs investigated.

\subsection{Shooting Method}

The equations of motion of system (1) can be recast into state space form

$$
\dot{\mathbf{z}}=\mathbf{g}(\mathbf{z})
$$

where $\mathbf{z}=\left[\begin{array}{ll}\mathbf{x}^{*} & \dot{\mathbf{x}}^{*}\end{array}\right]^{*}$ is the $2 n$-dimensional state vector, and star denotes the transpose operation, and

$$
\mathbf{g}(\mathbf{z})=\left(\begin{array}{c}
\dot{\mathbf{x}} \\
-\mathbf{M}^{-1}\left[\mathbf{K} \mathbf{x}+\mathbf{f}_{\mathrm{nl}}(\mathbf{x}, \dot{\mathbf{x}})\right]
\end{array}\right)
$$

is the vector field. The solution of this dynamical system for initial conditions $\mathbf{z}(0)=\mathbf{z}_{0}=\left[\begin{array}{ll}\mathbf{x}_{0}^{*} & \dot{\mathbf{x}}_{0}^{*}\end{array}\right]^{*}$ is written as $\mathbf{z}(t)=\mathbf{z}\left(t, \mathbf{z}_{0}\right)$ in order to exhibit the dependence on the initial conditions, $\mathbf{z}\left(0, \mathbf{z}_{0}\right)=\mathbf{z}_{0}$. A solution $\mathbf{z}_{p}\left(t, \mathbf{z}_{p 0}\right)$ is a periodic solution of the autonomous system (16) if $\mathbf{z}_{p}\left(t, \mathbf{z}_{p 0}\right)=\mathbf{z}_{p}\left(t+T, \mathbf{z}_{p 0}\right)$, where $T$ is the minimal period.

The NNM computation is carried out by finding the periodic solutions of the governing nonlinear equations of motion (16). In this context, the shooting method is probably the most popular numerical technique. It solves numerically the two-point boundary-value problem defined by the periodicity condition

$$
\mathbf{H}\left(\mathbf{z}_{p 0}, T\right) \equiv \mathbf{z}_{p}\left(T, \mathbf{z}_{p 0}\right)-\mathbf{z}_{p 0}=\mathbf{0}
$$

$\mathbf{H}\left(\mathbf{z}_{0}, T\right)=\mathbf{z}\left(T, \mathbf{z}_{0}\right)-\mathbf{z}_{0}$ is called the shooting function and represents the difference between the initial conditions and the system response at time $T$. Unlike forced motion, the period $T$ of the free response is not known a priori.

The shooting method consists in finding, in an iterative way, the initial conditions $\mathbf{z}_{p 0}$ and the period $T$ that realize a periodic motion. To this end, the method relies on direct numerical time integration and on the Newton-Raphson algorithm. 


\begin{tabular}{|c|c|c|}
\hline Model & Valid modes & Frequency range covered \\
\hline ROM84 & $1-18$ & {$[0-95.4] \mathrm{Hz}$} \\
ROM85 & $1-50$ & {$[0-248.2] \mathrm{Hz}$} \\
ROM86 & $1-246$ & {$[0-1020.8] \mathrm{Hz}$} \\
\hline
\end{tabular}

Table 4: Summary of the different ROMs performances.

Starting from some assumed initial conditions $\mathbf{z}_{p 0}^{(0)}$, the motion $\mathbf{z}_{p}^{(0)}\left(t, \mathbf{z}_{p 0}^{(0)}\right)$ at the assumed period $T^{(0)}$ can be obtained by numerical time integration methods (e.g., Runge-Kutta or Newmark schemes). In general, the initial guess $\left(\mathbf{z}_{p 0}^{(0)}, T^{(0)}\right)$ does not satisfy the periodicity condition (18). A Newton-Raphson iteration scheme is therefore to be used to correct an initial guess and to converge to the actual solution. The corrections $\Delta \mathbf{z}_{p 0}^{(k)}$ and $\Delta T^{(k)}$ at iteration $k$ are found by expanding the nonlinear function

$$
\mathbf{H}\left(\mathbf{z}_{p 0}^{(k)}+\Delta \mathbf{z}_{p 0}^{(k)}, T^{(k)}+\Delta T^{(k)}\right)=0
$$

in Taylor series and neglecting higher-order terms (H.O.T.).

The phase of the periodic solutions is not fixed. If $\mathbf{z}(t)$ is a solution of the autonomous system (16), then $\mathbf{z}(t+\Delta t)$ is geometrically the same solution in state space for any $\Delta t$. Hence, an additional condition, termed the phase condition, has to be specified in order to remove the arbitrariness of the initial conditions. This is discussed in detail in ${ }^{[7]}$.

In summary, an isolated NNM is computed by solving the augmented two-point boundary-value problem defined by

$$
\mathbf{F}\left(\mathbf{z}_{p 0}, T\right) \equiv\left\{\begin{array}{ccc}
\mathbf{H}\left(\mathbf{z}_{p 0}, T\right) & = & 0 \\
h\left(\mathbf{z}_{p 0}\right) & = & 0
\end{array}\right.
$$

where $h\left(\mathbf{z}_{p 0}\right)=0$ is the phase condition.

\subsection{Continuation of Periodic Solutions}

Due to the frequency-energy dependence, the modal parameters of an NNM vary with the total energy. An NNM family, governed by equations (20), therefore traces a curve, termed an NNM branch, in the $(2 n+1)$-dimensional space of initial conditions and period $\left(\mathbf{z}_{p 0}, T\right)$. Starting from the corresponding LNM at low energy, the computation is carried out by finding successive points $\left(\mathbf{z}_{p 0}, T\right)$ of the NNM branch using methods for the numerical continuation of periodic motions (also called path-following methods) $[8,9]$. The space $\left(\mathbf{z}_{p 0}, T\right)$ is termed the continuation space.

Different methods for numerical continuation have been proposed in the literature. The so-called pseudo-arclength continuation method is used herein.

Starting from a known solution $\left(\mathbf{z}_{p 0,(j)}, T_{(j)}\right)$, the next periodic solution $\left(\mathbf{z}_{p 0,(j+1)}, T_{(j+1)}\right)$ on the branch is computed using a predictor step and a corrector step.

\section{Predictor step}

At step $j$, a prediction $\left(\tilde{\mathbf{z}}_{p 0,(j+1)}, \tilde{T}_{(j+1)}\right)$ of the next solution $\left(\mathbf{z}_{p 0,(j+1)}, T_{(j+1)}\right)$ is generated along the tangent vector to the branch at the current point $\mathbf{z}_{p 0,(j)}$

$$
\left[\begin{array}{c}
\tilde{\mathbf{z}}_{p 0,(j+1)} \\
\tilde{T}_{(j+1)}
\end{array}\right]=\left[\begin{array}{c}
\mathbf{z}_{p 0,(j)} \\
T_{(j)}
\end{array}\right]+s_{(j)}\left[\begin{array}{c}
\mathbf{p}_{z,(j)} \\
p_{T,(j)}
\end{array}\right]
$$

where $s_{(j)}$ is the predictor stepsize. The tangent vector $\mathbf{p}_{(j)}=\left[\mathbf{p}_{z,(j)}^{*} p_{T,(j)}\right]^{*}$ to the branch defined by $(20)$ is solution of the system

$$
\left[\begin{array}{c}
\left.\frac{\partial \mathbf{H}}{\partial \mathbf{z}_{p 0}}\right|_{\left(\mathbf{z}_{p 0,(j)}, T_{(j)}\right)} \\
\left.\frac{\partial h}{\partial \mathbf{z}_{p 0}}\right|_{\left(\mathbf{z}_{p 0,(j)}\right)}
\end{array}\right]\left[\begin{array}{l}
\mathbf{p}_{z,(j)} \\
p_{T,(j)}
\end{array}\right]=\left[\begin{array}{l}
\mathbf{0} \\
0
\end{array}\right]
$$


with the condition $\left\|\mathbf{p}_{(j)}\right\|=1$. The star denotes the transpose operator. This normalization can be taken into account by fixing one component of the tangent vector and solving the resulting overdetermined system using the Moore-Penrose matrix inverse; the tangent vector is then normalized to 1 .

\section{Corrector step}

The prediction is corrected by a shooting procedure in order to solve (20) in which the variations of the initial conditions and the period are forced to be orthogonal to the predictor step. At iteration $k$, the corrections

$$
\begin{aligned}
\mathbf{z}_{p 0,(j+1)}^{(k+1)} & =\mathbf{z}_{p 0,(j+1)}^{(k)}+\Delta \mathbf{z}_{p 0,(j+1)}^{(k)} \\
T_{(j+1)}^{(k+1)} & =T_{(j+1)}^{(k)}+\Delta T_{(j+1)}^{(k)}
\end{aligned}
$$

are computed by solving the overdetermined linear system using the Moore-Penrose matrix inverse

$$
\left[\begin{array}{c}
\left.\frac{\partial \mathbf{H}}{\partial \mathbf{z}_{p 0}}\right|_{\left(\mathbf{z}_{p 0,(j+1)}^{(k)}, T_{(j+1)}^{(k)}\right)} \\
\left.\frac{\partial \mathbf{H}^{*}}{\partial T}\right|_{\left(\mathbf{z}_{p 0,(j+1)}^{(k)}, T_{(j+1)}^{(k)}\right)} \\
\mathbf{p}_{z,(j)}^{*}
\end{array}\right]\left[\begin{array}{c}
\Delta \mathbf{z}_{p 0,(j+1)}^{(k)} \\
\Delta T_{(j+1)}^{(k)}
\end{array}\right]=\left[\begin{array}{c}
-\mathbf{H}\left(\mathbf{z}_{p 0,(j+1)}^{(k)}, T_{(j+1)}^{(k)}\right) \\
-h\left(\mathbf{z}_{p 0,(j+1)}^{(k)}\right) \\
0
\end{array}\right]
$$

where the prediction is used as initial guess, i.e, $\mathbf{z}_{p 0,(j+1)}^{(0)}=\tilde{\mathbf{z}}_{p 0,(j+1)}$ and $T_{(j+1)}^{(0)}=\tilde{T}_{(j+1)}$. The last equation in (24) corresponds to the orthogonality condition for the corrector step.

This iterative process is carried out until convergence is achieved. The convergence test is based on the relative error of the periodicity condition:

$$
\frac{\left\|\mathbf{H}\left(\mathbf{z}_{p 0}, T\right)\right\|}{\left\|\mathbf{z}_{p 0}\right\|}=\frac{\left\|\mathbf{z}_{p}\left(T, \mathbf{z}_{p 0}\right)-\mathbf{z}_{p 0}\right\|}{\left\|\mathbf{z}_{p 0}\right\|}<\epsilon
$$

where $\epsilon$ is the prescribed relative precision.

\subsection{Sensitivity Analysis}

Each shooting iteration involves the time integration of the equations of motion to evaluate the current shooting residue $\mathbf{H}\left(\mathbf{z}_{p 0}^{(k)}, T^{(k)}\right)=$ $\mathbf{z}_{p}^{(k)}\left(T^{(k)}, \mathbf{z}_{p 0}^{(k)}\right)-\mathbf{z}_{p 0}^{(k)}$. As evidenced by equation (24), the method also requires the evaluation of the $2 n \times 2 n$ Jacobian matrix

$$
\frac{\partial \mathbf{H}}{\partial \mathbf{z}_{0}}\left(\mathbf{z}_{0}, T\right)=\left.\frac{\partial \mathbf{z}\left(t, \mathbf{z}_{0}\right)}{\partial \mathbf{z}_{0}}\right|_{t=T}-\mathbf{I}
$$

where $\mathbf{I}$ is the $2 n \times 2 n$ identity matrix.

The classical finite-difference approach requires to perturb successively each of the $2 n$ initial conditions and integrate the nonlinear governing equations of motion. This approximate method therefore relies on extensive numerical simulations and may be computationally intensive for large-scale finite element models.

Targeting a reduction of the computational cost, a significant improvement is to use sensitivity analysis for determining $\partial \mathbf{z}\left(t, \mathbf{z}_{0}\right) / \partial \mathbf{z}_{0}$ instead of a numerical finite-difference procedure. The sensitivity analysis consists in differentiating the equations of motion (16) with respect to the initial conditions $\mathbf{z}_{0}$ which leads to

$$
\frac{d}{d t}\left[\frac{\partial \mathbf{z}\left(t, \mathbf{z}_{0}\right)}{\partial \mathbf{z}_{0}}\right]=\left.\frac{\partial \mathbf{g}(\mathbf{z})}{\partial \mathbf{z}}\right|_{\mathbf{z}\left(t, \mathbf{z}_{0}\right)}\left[\frac{\partial \mathbf{z}\left(t, \mathbf{z}_{0}\right)}{\partial \mathbf{z}_{0}}\right]
$$

with

$$
\frac{\partial \mathbf{z}\left(0, \mathbf{z}_{0}\right)}{\partial \mathbf{z}_{0}}=\mathbf{I}
$$

since $\mathbf{z}\left(0, \mathbf{z}_{0}\right)=\mathbf{z}_{0}$. Hence, the matrix $\partial \mathbf{z}\left(t, \mathbf{z}_{0}\right) / \partial \mathbf{z}_{0}$ at $t=T$ can be obtained by numerically integrating over $T$ the initial-value problem defined by the linear ordinary differential equations (ODEs) (27) with the initial conditions (28). 


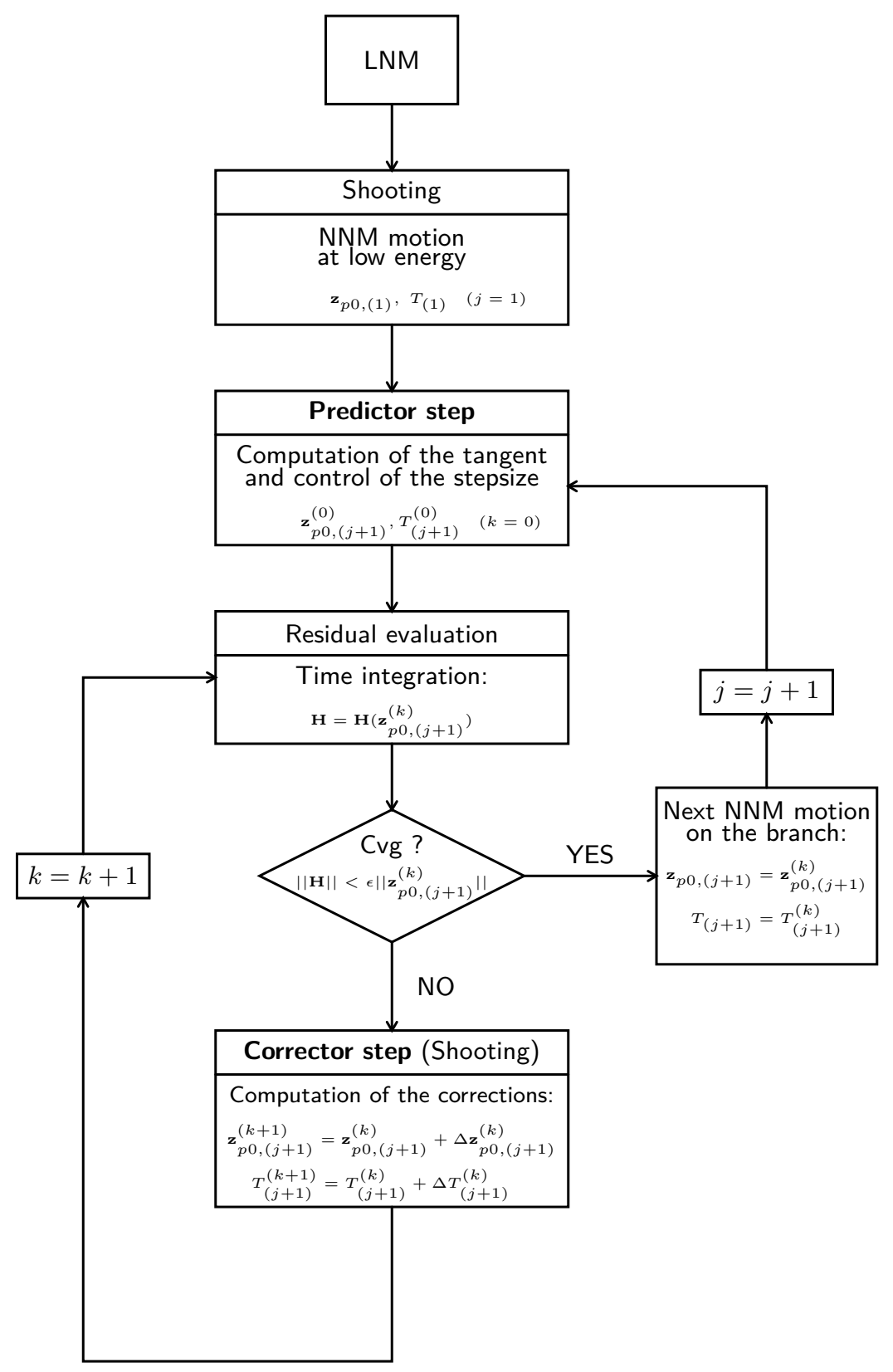

Figure 9: Algorithm for NNM computation. 
In addition to the integration of the current solution $\mathbf{z}\left(t, \mathbf{x}_{0}\right)$ of (16), these two methods for computing $\partial \mathbf{z}\left(t, \mathbf{z}_{0}\right) / \partial \mathbf{z}_{0}$ require $2 n$ numerical integrations of $2 n$-dimensional dynamical systems, which may be computationally intensive for large systems. However, equations (27) are linear ODEs and their numerical integration is thus less expensive. The numerical cost can be further reduced if the solution of equations (27) is computed together with the solution of the nonlinear equations of motion in a single numerical simulation ${ }^{[10]}$.

The sensitivity analysis requires only one additional iteration at each time step of the numerical time integration of the current motion to provide the Jacobian matrix. The reduction of the computational cost is therefore significant for large-scale finite element models. In addition, the Jacobian computation by means of the sensitivity analysis is exact. The convergence troubles regarding the chosen perturbations of the finite-difference method are then avoided. Hence, the use of sensitivity analysis to perform the shooting procedure represents a meaningful improvement from a computational point of view.

As the monodromy matrix $\partial \mathbf{z}_{p}\left(T, \mathbf{z}_{p 0}\right) / \partial \mathbf{z}_{p 0}$ is computed, its eigenvalues, the Floquet multipliers, are obtained as a by-product, and the stability analysis of the NNM motions can be performed in a straightforward manner.

\subsection{Algorithm for NNM Computation}

The algorithm proposed for the computation of NNM motions is a combination of shooting and pseudo-arclength continuation methods, as shown in Figure 9. It has been implemented in the MATLAB environment. Other features of the algorithm such as the step control, the reduction of the computational burden and the method used for numerical integration of the equations of motion are discussed in ${ }^{[7]}$.

So far, the NNMs have been considered as branches in the continuation space $\left(\mathbf{z}_{p 0}, T\right)$. An appropriate graphical depiction of the NNMs is to represent them in a frequency-energy plot (FEP). This FEP can be computed in a straightforward manner: (i) the conserved total energy is computed from the initial conditions realizing the NNM motion; and (ii) the frequency of the NNM motion is calculated directly from the period.

\section{NONLINEAR MODAL ANALYSIS}

As presented in Section 3, the nonlinearities are located at WEMS ends. These nonlinearities are activated when displacements are large enough to hit the mechanical stops. Due to the flexibility of WEMS attachment, large displacements are observed for the majority of LNMs. However, according to the linear study of Section 4, the nonlinear investigations are restricted to four modes.

The energy range of interest for the continuation is determined by the displacements observed at WEMS ends. Indeed, experimental observation demonstrated that displacements are limited. Therefore, relative displacements larger than the observed values are not representative of the real physical behavior of the structure.

Among the NNMs presented, one can readily distinguish two categories of modes, namely the energy-dependent (e.g. Figure 11) and the energy-independent modes (e.g., Figure 10). The latter correspond to the nonlinear extension of linear modes that do not involve WEMS motion. An example is provided by the ninth mode (Figure 10) where the deformation is localized at the SASSA. As the energy increases, the modal shape (Figure $5(d)$ ) presented in the linear study is unchanged and the mode remains linear.

Figure 11 presents the continuation of the first LNM. This LNM correspond to a local WEMS motion and is therefore sensitive to nonlinearities present in the model. For low energies, the structural behavior remains purely linear and the resonance frequency does not depend on the energy. However, as energy increases, a steep modification of the frequency-energy dependence appears. At this transition, one can observe that relative displacements at WEMS ends enter in the regularization area. Beyond this transition, a plateau appears and interactions between the first LNM and other LNMs are achieved. The frequency content of the periodic solution evolves with the energy and includes third, fifth, and higher-order harmonics (up to the $17^{\text {th }}$ order).

Figure 12 displays the frequency-energy dependence of the third NNM. It highlights the presence of a tongue, revealing the existence 


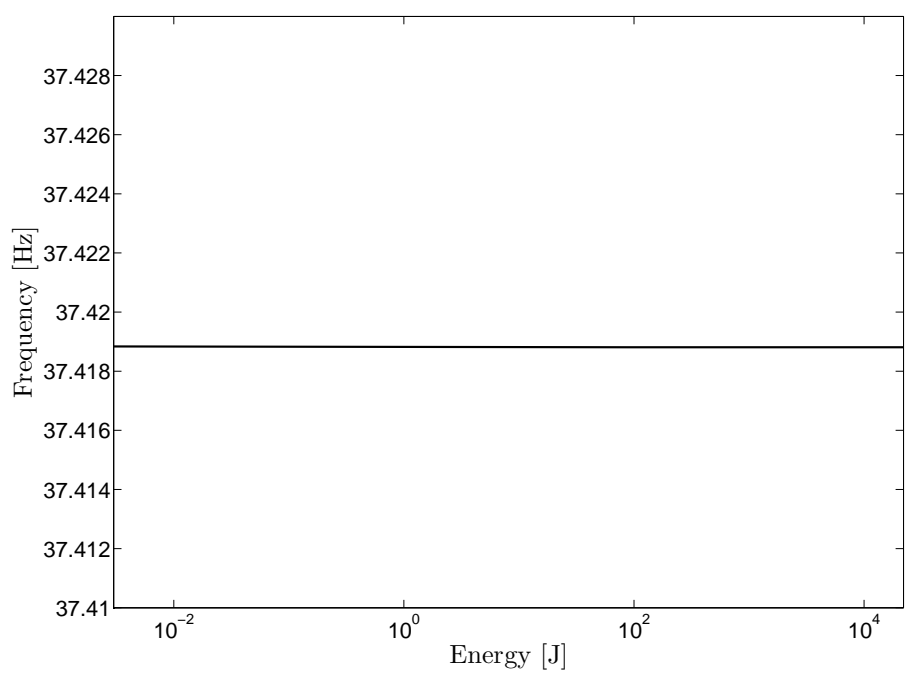

Figure 10: Frequency-energy dependence of the ninth NNM.

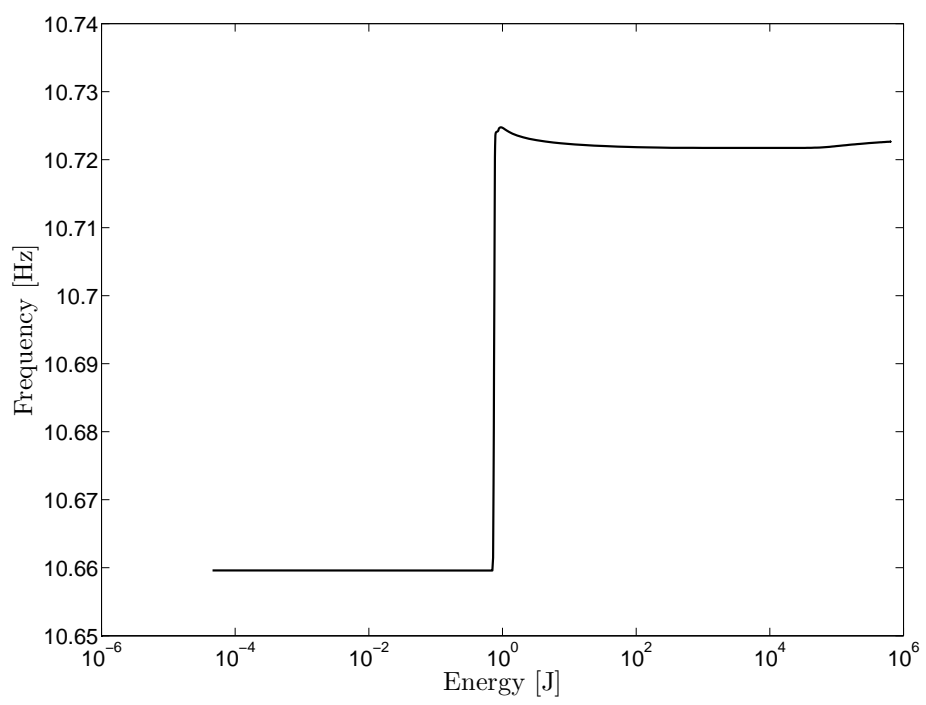

Figure 11: Frequency-energy dependence of the first NNM.

of an $3: 1$ internal resonance between the third (Figure 5(b)) and the sixteenth LNM (Figure 13(b)). The latter corresponds to an octagonal structure panels mode. Along the tongue (e.g., point (b) in Figure 12), the modal shape evolves from the third to the sixteenth LNM and is therefore a special combination of both modes (Figure 13(a)). This mode has no linear counterpart and highlights the possibility of interactions between local and global SC modes due to the presence of nonlinearities.

The frequency-energy dependence of the seventh NNM is presented in Figure 14. The presence of a tongue again highlights modal interactions between the seventh LNM (Figure 5(c)) and a higher-order LNM. Here, it is interesting to observe that the seventh LNM mainly involves a motion of the SASSA. However, due to the nonlinearities of the WEMS, nonlinear couplings between this SASSA mode and global structural modes are achieved. 


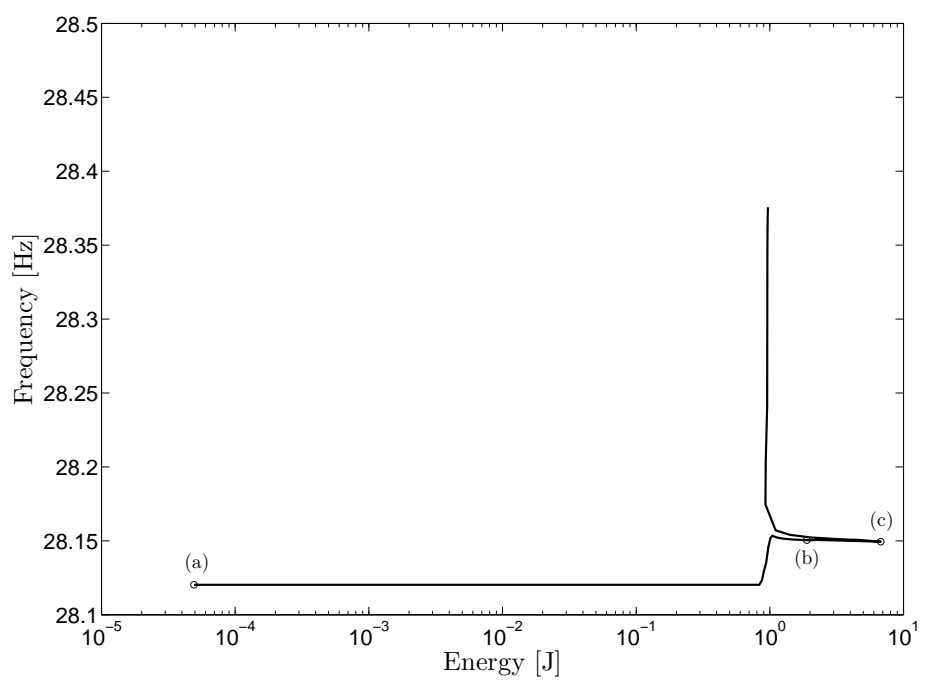

Figure 12: Frequency-energy dependence of the third NNM. (a) Low-energy point. (b) Point in the tongue describing the $3: 1$ internal resonance. (c) Bifurcation point.

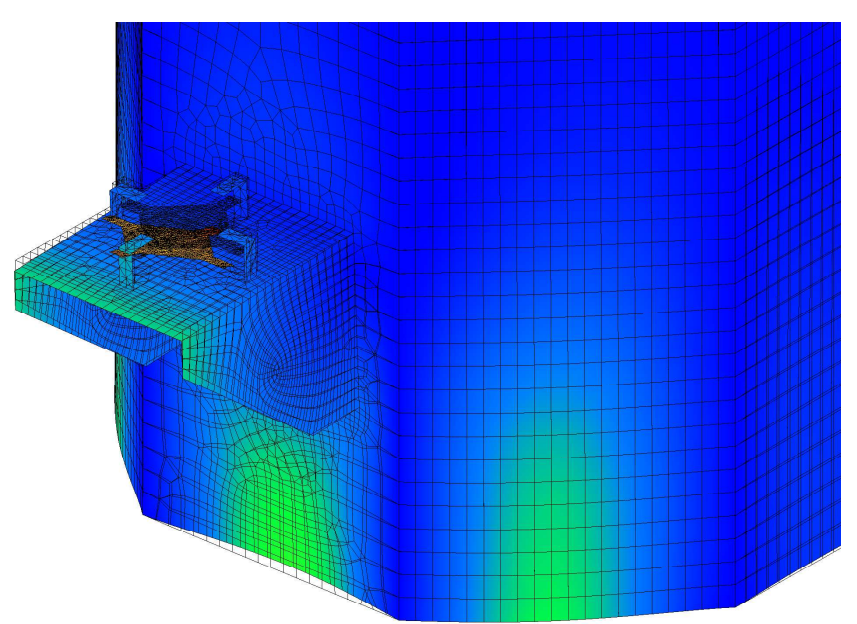

(a)

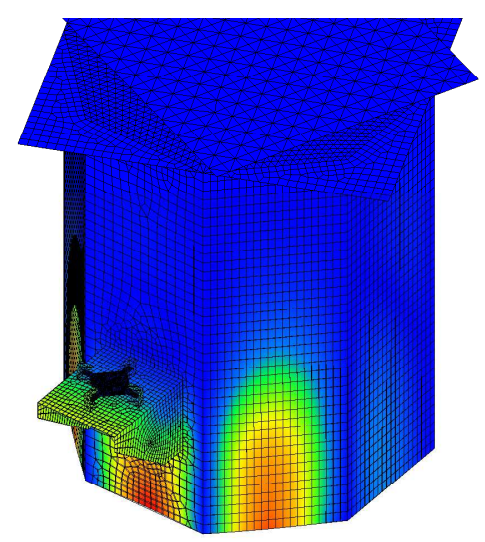

(b)

Figure 13: (a) Modal shape of the third NNM combining the third and the sixteenth LNMs ((b) in Figure 12). (b) Modal shape at the internal resonance equivalent to the sixteenth LNM (WEMS remains quiescent)((c) in Figure 12).

\section{CONCLUSIONS}

In this paper, the fundamental concepts regarding undamped nonlinear normal modes and their numerical computation were reviewed. A new regularization procedure was presented and revealed to be accurate for the modeling of piecewise linear restoring forces.

Targeting the computation of the nonlinear modes, a linear modal analysis was presented and some interesting modes were identified for further investigations. A reduced-order model accurate in the $[0-200 \mathrm{~Hz}]$ range was employed to reduce the computational burden.

Finally, the nonlinear normal modes of the spacecraft were presented. Internal resonances highlighted the possibility of mode interactions between local (WEMS) and global structural modes. 


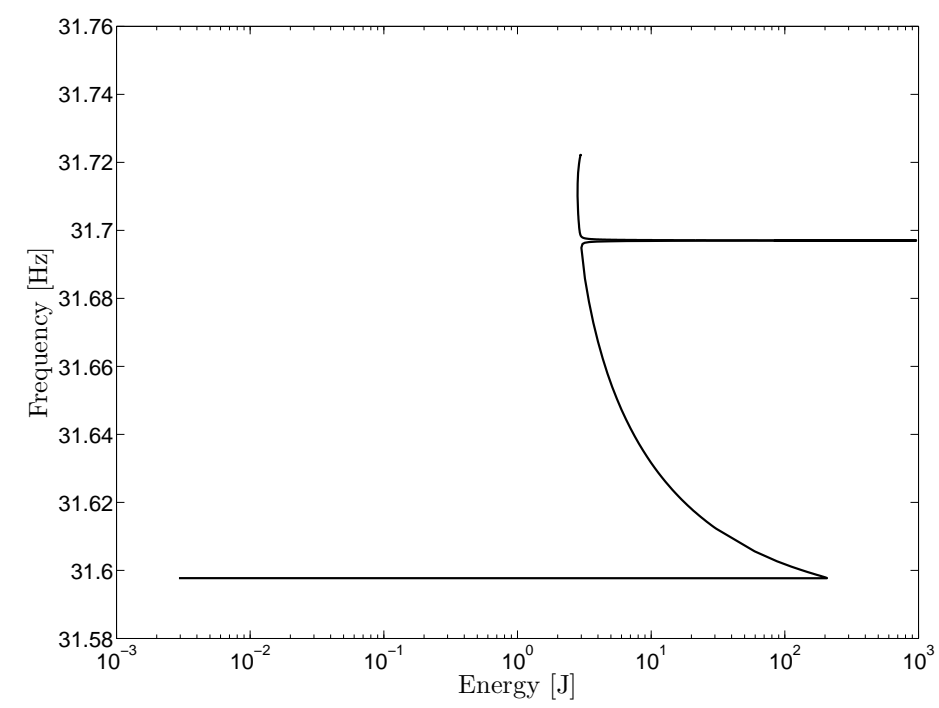

Figure 14: Frequency-energy dependence of the seventh NNM.

\section{ACKNOWLEDGMENTS}

This paper has been prepared in the framework of the ESA Technology Research Programme study "Advancement of Mechanical Verification Methods for Non-linear Spacecraft Structures (NOLISS)" (ESA contract No.21359/08/NL/SFe).

The authors would like to thank Dr. Maxime Peeters for all the constructive discussions. The author L. Renson would like to acknowledge the Belgian National Fund for Scientific Research (FRIA fellowship) for its financial support.

\section{References}

[1] A.F. Vakakis, L.I. Manevitch, Y.V. Mikhlin, V.N. Pilipchuk, A.A. Zevin, Normal Modes and Localization in Nonlinear Systems, John Wiley \& Sons, New York (1996).

[2] G. Kerschen, M. Peeters, J.C. Golinval, A.F. Vakakis, Nonlinear normal modes, Part I: A useful framework for the structural dynamicist, Mechanical Systems and Signal Processing, Vol. 23, No. 1 (2009), pp. 170-194.

[3] Y.S. Lee, G. Kerschen, A.F. Vakakis, P.N. Panagopoulos, L.A. Bergman, D.M. McFarland, Complicated dynamics of a linear oscillator with a light, essentially nonlinear attachment, Physica D-Nonlinear Phenomena, Vol. 204, No. 1-2 (2005), pp. 41-69.

[4] A. G. Russell Thick skin, faceted, CFRP, monocoque tube structure for smallsats, European Conference on Spacecraft Structures, Materials and Mechanical Testing (2000).

[5] P. Camarasa and S. Kiryenko, Shock attenuation system for spacecraft and adaptor (SASSA), European Conference on Spacecraft Structures, Materials and Mechanical Testing (2009).

[6] R. Craig, M. Bampton, Coupling of substructures for dynamic analysis, AIAA Journal, Vol. 6 (1968), pp. $1313-1319$.

[7] M. Peeters, R. Viguié, G. Sérandour, G. Kerschen, J.C. Golinval, Nonlinear normal modes, Part II: Toward a practical computation using numerical continuation techniques, Mechanical Systems and Signal Processing, Vol. 23, No. 1 (2009), pp. 195-216.

[8] R. Seydel, Practical Bifurcation and Stability Analysis, from Equilibirum to Chaos, Springer-Verlag, 2nd Edition (1994). 
[9] A.H. Nayfeh, B. Balachandran, Applied Nonlinear Dynamics: Analytical, Computational, and Experimental Methods, WileyInterscience, New York (1995).

[10] O. Brüls, P. Eberhard, Sensitivity analysis for dynamic mechanical systems with finite rotations, International Journal for Numerical Methods in Engineering, Vol. 1 (2006), pp. 1-29. 